\title{
Diabetic retinopathy screening in urban primary care setting with a handheld smartphone-based retinal camera
}

\author{
Márcia Silva Queiroz ${ }^{1}$. Jacira Xavier de Carvalho ${ }^{2}$. Silvia Ferreira Bortoto ${ }^{1} \cdot$ Mozania Reis de Matos $^{2}$. \\ Cristiane das Graças Dias Cavalcante ${ }^{2}$ - Elenilda Almeida Silva Andrade ${ }^{2} \cdot$ Maria Lúcia Correa-Giannella $^{1}$. \\ Fernando Korn Malerbi ${ }^{3,4}$ [i]
}

Received: 5 June 2020 / Accepted: 21 July 2020 / Published online: 4 August 2020

(c) Springer-Verlag Italia S.r.l., part of Springer Nature 2020

\begin{abstract}
Aims To evaluate diabetic retinopathy (DR) screening with a portable handheld smartphone-based retinal camera and telemedicine in an urban primary healthcare setting and to evaluate the learning curve for image acquisition, performed by healthcare personnel without previous experience in retinal imaging.

Methods This was a prospective study that enrolled patients with type 2 diabetes mellitus (T2DM) followed at a primary healthcare unit in São Paulo, Brazil. After a brief training in image acquisition, there was further continuous feedback given by a retina specialist during the remote image reading process. Each patient underwent two fundus and one anterior ocular segment images per eye, after mydriasis. Patients were classified according to the need of referral.

Results A total of 627 adult individuals with T2DM underwent retinal evaluation. The population was composed by $63.2 \%$ female individuals, age median of 66 years, diabetes duration $10.7 \pm 8.2$ years and $\mathrm{HbA} 1 \mathrm{c} 7.7 \pm 1.9 \%(61+20.8 \mathrm{mmol} / \mathrm{mol})$. The most prevalent associated comorbidities were arterial hypertension (80.3\%) and dyslipidemia (50.2\%). Referral decision was possible in $81.2 \%$ patients. Most patients had absent or non-referable DR; the main ocular media opacity detected was cataract. After the 7th day of image acquisition, the daily rate of patients whose images allowed clinical decision was maintained above $80 \%$. A higher HbA1c was associated with referable DR.

Conclusions A low-cost DR screening strategy with a handheld device and telemedicine is feasible and has the potential to increase coverage of DR screening in underserved areas; the possibility of mobile units is relevant for DR screening in the context of COVID-19 pandemic.
\end{abstract}

This article belongs to the topical collection Eye Complications of Diabetes, managed by Giuseppe Querques.

Fernando Korn Malerbi

fernandokmalerbi@gmail.com

1 Programa de Pos-Graduaçao em Medicina, Universidade Nove de Julho (UNINOVE), Rua Vergueiro 235, $2^{\circ}$ subsolo, Pos-graduação, Sao Paulo 01504-001, Brazil

2 Unidade Basica de Saude Dra. Ilza Weltman Hutzler, Rua Coronel Walfrido de Carvalho, Sao Paulo 02472-180, Brazil

3 Programa de Graduaçao em Medicina, Universidade Nove de Julho (UNINOVE), Rua Vergueiro 235, Sao Paulo 01504-001, Brazil

4 Departamento de Oftalmologia, Universidade Federal de São Paulo, Rua Botucatu 822, São Paulo 04039-032, Brazil 


\section{Graphic abstract}

Daily rate of patients whose examinations allowed clinical decision. $X$-axis: day of examination; $Y$-axis: rate (\%) of patients whose examinations allowed a clinical decision

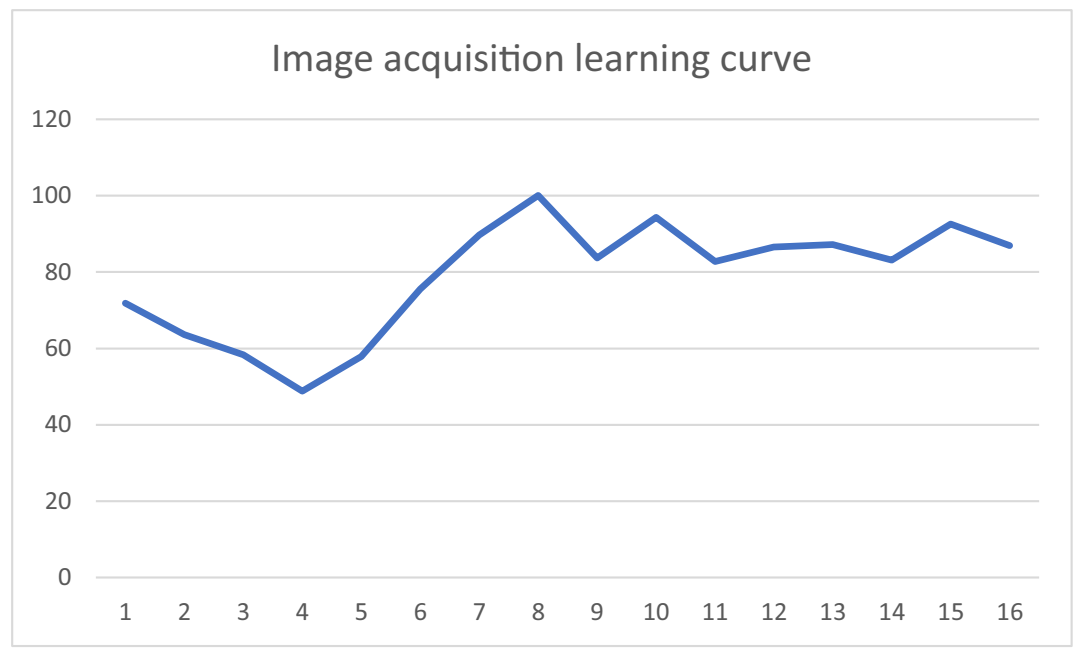

$X$ axis: day of exam; $Y$ axis: rate (\%) of patients whose exams allowed a clinical decision

Keywords Diabetic retinopathy $\cdot$ Telemedicine $\cdot$ Blindness $\cdot$ Screening $\cdot$ Primary care $\cdot$ COVID-19

\section{Introduction}

Diabetic retinopathy (DR) is a leading cause of severe visual loss which affects approximately 30 million individuals worldwide and can be prevented with timely screening, detection and treatment; however, even in high-income countries, access to yearly eye examinations is limited to only one-third to one-half of adults with diabetes, leaving a substantial number of patients at risk [1]. In the USA, only about $60 \%$ of people with diabetes have recommended yearly screenings for diabetic retinopathy; in some community-based studies, that rate is even lower [2]; in middle- to low-income countries, where access to ophthalmic care is generally limited, the rate of individuals with diabetes who have access to eye examinations is remarkably low [3]. In Brazil, primary health care is developed through forms of teamwork composed of a physician, a nurse, nursing assistants and community agents of health, aiming to assist populations defined by basic health units ("Unidade Básica de Saúde"—UBSs). In turn, the UBS is responsible for support and infrastructure; since there is no availability of an ophthalmologist in the medical staff of most UBSs, diabetic retinopathy (DR) screening is performed in secondary care units [4]; hence, the waiting time for an appointment is often increased, causing DR monitoring and timely treatment to be extremely difficult.
Teleophthalmology programs have facilitated the identification of DR cases by utilizing digital retinal imaging [5]; additionally, teleophthalmology based on mobile imaging units can ameliorate DR screening and decrease the possibility of poor compliance or lost follow-up appointments [6]. The combination of teleophthalmology with portable devices may further increase access to eye care in underserved populations. The importance of the teleophthalmology strategy lies on decreasing the burden on the health system, referring to specialized care only those individuals with treatable conditions and allowing timely treatment to prevent diabetic blindness. Hence, image quality is paramount to the success of the screening program, as low-quality images will not yield diagnosis, making necessary an evaluation by the specialist.

Handheld cameras are increasingly available for diabetic retinopathy screening, having several advantages over traditional tabletop cameras, such as increased portability and decreased cost, with the potential of improving DR screening rates [1]. For optimal image quality, training in image acquisition and ocular media transparence are needed.

The present study was designed to evaluate diabetic retinopathy screening with a portable retinal camera and telemedicine in a primary healthcare urban setting. The learning curve for image acquisition, performed by non-medical healthcare personnel without previous experience on retinal imaging, was also evaluated. 


\section{Materials and methods}

This study enrolled 627 individuals aged over 18 years with a previous type 2 diabetes mellitus (T2DM) diagnosis who were treated in a UBS on the outskirts of the city of São Paulo. Non-inclusion criteria were not adopted. The study was conducted in compliance with the Declaration of Helsinki, in accordance with the institutional ethics committees (\#3050387 and \#3141417). After being informed of the risks of pupil dilation, generally consisting of transient ocular discomfort, blurry vision and photophobia, participants signed the informed consent, were assessed for demographic, clinical and biochemical features and had the anterior and posterior ocular segments of both eyes photographed after mydriasis (Fig. 1).

Clinical and demographic data analysis shows that the population was composed by $63.2 \%$ female individuals, age median of 66 years, diabetes duration $10.7 \pm 8.2$ years and $\mathrm{HbA} 1 \mathrm{c} 7.7 \pm 1.9 \%(61+20.8 \mathrm{mmol} / \mathrm{mol})$. The most prevalent associated comorbidities were arterial hypertension (80.3\%) and dyslipidemia (50.2\%).

A smartphone-based handheld device (Eyer, Phelcom Technologies, São Carlos, Brazil) was used for retinal image acquisition according to a previous protocol used in the Brazilian multicenter study of diabetic retinopathy [7], and photographs of the anterior and posterior ocular segments were obtained for both eyes, after mydriasis induced by two $1 \%$ tropicamide eye drops. Two $45^{\circ}$ images of the posterior segment, one field centered on the fovea and the other field centered on the optic disk of each fundus, were captured. The professionals responsible for image acquisition were a team of four nurses involved in diabetes care in their own basic health unit, without previous experience in this kind of procedure. They underwent a four-hour training about image protocol and acquisition procedure by an ophthalmologist, and thereafter, they started performing retinal imaging and relied on the continuous remote feedback given by the specialist as images were interpreted. Image acquisition occurred from February 6 to March 14, 2020 , comprising a 5-week period, in non-consecutive days.

Remote image reading was performed in a store-andforward approach at EyerCloud platform (Phelcom Technologies, São Carlos, Brazil) by the same retinal specialist (FKM). First, the photographs of patients' retinas were evaluated by quality and classified as gradable or ungradable images. Subsequently, diabetic retinopathy classification was performed for those with gradable images, and patients with ungradable images had their anterior ocular segment images assessed regarding the presence of cataracts or other media opacities. Whenever the severity of cataract or of other media opacity precluded fundus evaluation, the patient would be considered as referable for ophthalmologic evaluation. When ocular media had enough transparence to allow DR grading, each patient was categorized according to the most affected eye, in accordance with the American Diabetes Association criteria [8]: non-referable (absent or mild to moderate non-proliferative diabetic retinopathy without diabetic maculopathy) or referable (severe non-proliferative diabetic retinopathy; proliferative diabetic retinopathy; presence of macular edema in at least one eye). Table 1 presents the retinal lesions found at each DR classification level [7]. The images with poor quality for reasons other than media opacities and also images from patients who did not comply with the proposed protocol, for example, by lack of image fields or image taken of non-representative fields, were considered as protocol failure; such patients were also referred for ophthalmological evaluation.
Fig. 1 Ocular images of screened individuals with diabetes. Left: Anterior segment image showing cataract. Right: Retinal photograph of the posterior pole of a patient with diabetes depicting microaneurysms, retinal hemorrhages, cotton-wool spots and hard exudates on the macular area

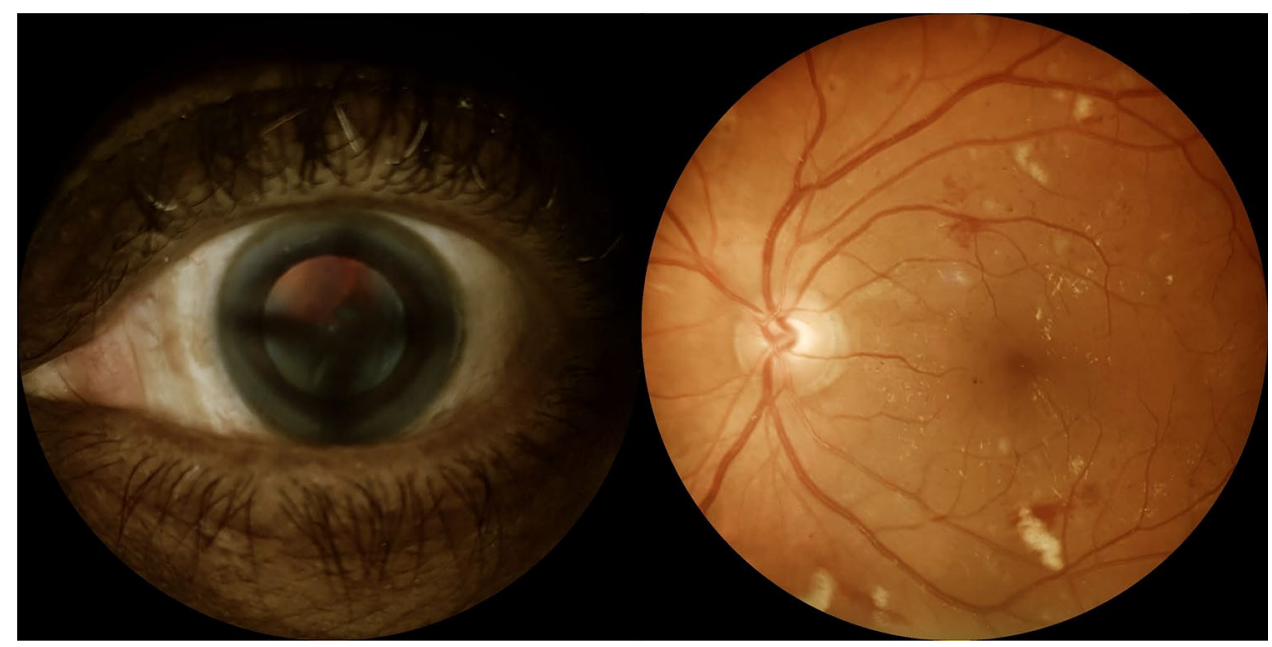


Table 1 Diabetic retinopathy severity levels

\begin{tabular}{ll}
\hline Severity level & Lesions \\
\hline Absent & No alterations \\
Mild non-proliferative & At least one hemorrhage or microaneurysm \\
Moderate non-proliferative & Four or more hemorrhages in only one hemi-field ${ }^{\mathrm{a}}$ \\
Severe non-proliferative & Any of the following: \\
& Four or more hemorrhages in the superior and \\
& inferior hemi-fields \\
& Venous beading \\
Proliferative & Intraretinal microvascular abnormalities (IRMA) \\
& Any of the following: \\
& Active neovessels \\
& Vitreous hemorrhage \\
\hline
\end{tabular}

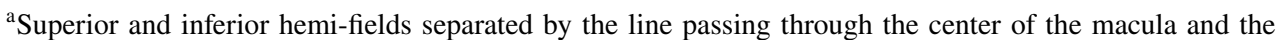
optic disk
To evaluate the image acquisition learning curve, a specific analysis was performed regarding each day of examination, and the daily rate of patients successfully evaluated by teleophthalmology, regarding the presence of media opacities, DR classification and presence of maculopathy, was calculated. Furthermore, information concerning the duration of examination per patient was collected.

\section{Statistical analysis}

Statistical analyses were performed using the SAS/STAT software. An ANOVA one-way model and Fisher's exact test were applied to compare variables, and the nonparametric Kruskal-Wallis test was employed for the comparison between patients' groups; $p$ values $<0.05$ were considered statistically significant.

\section{Results}

Six hundred and twenty-seven individuals with T2DM underwent retinal evaluation by the smartphone-based handheld device. The photographs allowed a clinical decision in 509 (81.2\%) patients, who either had their diabetic retinopathy graded (439) or presented with ocular media opacities that supported their referral to the specialist (70). In the remaining 118 patients (18.8\%), images were ungradable for causes other than ocular media opacities, such as inadequate technique or frames that did not comply with the proposed protocol; those patients were referred. Patients who were referred because of media opacities comprised 61 cases of cataracts and 9 cases of vitreous, corneal or posterior capsule opacities. Among the 439 gradable patients, DR classification was as follows: 333 with no DR, 40 with nonreferable DR and 66 with referable DR. Overall, a total of
373 patients had no indication for specialist referral because of DR and/or ocular media opacities (Fig. 2). There was no adverse reaction to mydriatic drops.

Except for HbA1c, all other clinical and demographic variables were not different regarding DR classification, as shown in Table 2.

The learning curve of image acquisition was evaluated according to the rate of patients whose images allowed clinical decision on daily basis. All images for the present study were collected along 16 non-consecutive days; from the 7 th day onward, the rate of patients whose images allowed clinical decision was maintained above $80 \%$, as shown in Graphic 1. Examination duration per patient had an average time of $2.5+1.7 \mathrm{~min}$ (median 2.0, maximum $15 \mathrm{~min}$ ).

In a sub-analysis, the patients were divided into two groups: those in whom DR could be graded or who had media opacities detected by the examination (images allowed a clinical decision) and those whose images were ungradable for causes other than ocular media opacities; clinical and technical variables were compared between these groups. Patients whose images did not yield were older $(p=0.04)$, and examination duration for image acquisition was longer $(p<0.001)$; other parameters such as gender, HbAlc and duration of diabetes did not reach statistical significance.

\section{Discussion}

After a brief training and a short learning curve, healthcare professionals without previous experience on retinal imaging acquisition performed well, attaining after 7 days a rate of over $80 \%$ of examined patients whose examination allowed clinical decision. The training, the image protocol and the remote reading, together with a short examination time, showed that image acquisition with a portable device associated with teleophthalmology is a feasible strategy for 


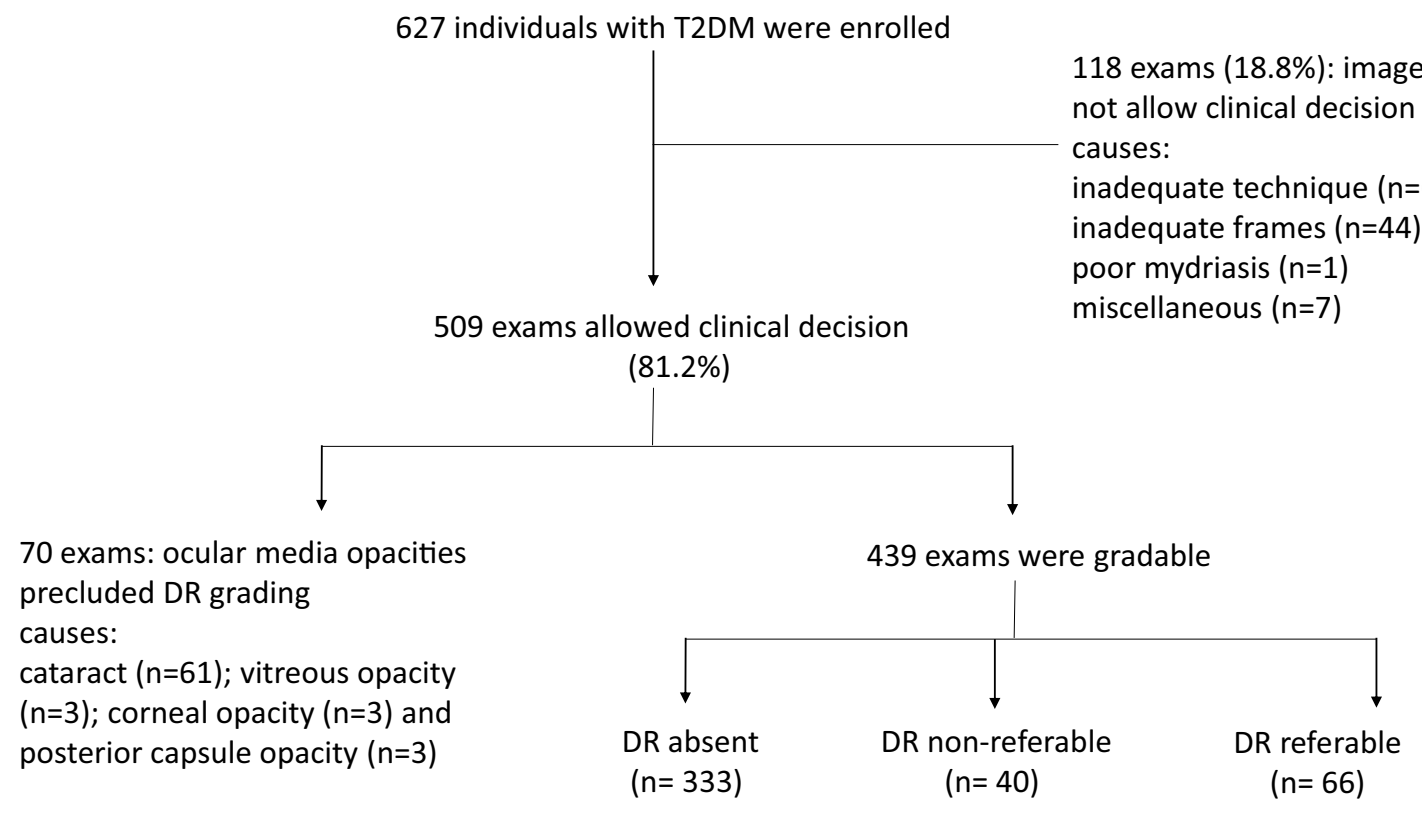

Fig. 2 Schematic flowchart of the study showing number of patients enrolled, decision tree and outcomes. T2DM: type 2 diabetes mellitus, n: number of patients; DR: diabetic retinopathy

Table 2 Clinical and demographic variables among patients classified as having DR absent, DR non-referable and DR referable

\begin{tabular}{llll}
\hline & DR absent & DR non-referable & DR referable \\
\hline Age (years) & $65.1 \pm 11.5$ & $66.5 \pm 9.4$ & $63.1 \pm 12.2$ \\
Gender M/F (\%) & $36.5 / 63.5$ & $23.8 / 76.2$ & $46 / 54$ \\
DM duration (years) & $10.8 \pm 8.4$ & $9.3 \pm 6.7$ & $10.8 \pm 7.5$ \\
HbA1c (\%) & $7.6 \pm 1.7$ & $7.4 \pm 1.4^{*}$ & $9.2 \pm 2.4^{\# \&}$ \\
HbA1c (mmol/mol) & $60 \pm 18.6$ & $57 \pm 15.3^{*}$ & $77 \pm 26.2^{\# \&}$ \\
Hypertension (\%) & 80.9 & 80.9 & 76.5 \\
Dyslipidemia (\%) & 49 & 52.7 & 56.2 \\
\hline
\end{tabular}

Data showed as mean \pm standard deviation; DR: diabetic retinopathy, M: male; F: female; DM: diabetes mellitus; HbA1c: glycated hemoglobin; DR: diabetic retinopathy; *DR absent compared to DR non-referable: $p=0.914,{ }^{\#} \mathrm{DR}$ absent compared to DR referable: $p=0.0062 ;{ }^{\&} \mathrm{DR}$ non-referable compared to DR referable: $p=0.0335$; ANOVA

DR and cataract screening in patients with diabetes who dwell in an urban area and are followed in a primary healthcare setting.

DR screening programs have been implemented with success over the last decades in several countries, resulting in lower rates of diabetic blindness [9-13]. Adequate image quality is a major factor upon which the success of the screening strategy is dependent [14], and we hereby present favorable results and a high rate of clinical yield attained after a brief training and subsequent continuous remote feedback. Of note is the previous lack of experience of the trained team, as previous reports have presented high-quality rates obtained with experienced personnel $[1,15,16]$. The sustainability and cost-effectiveness of screening programs are also fundamental for its implementation and success; to the best of our knowledge, ours is the first report on the performance of low-cost, portable handheld devices for DR screening in an urban setting in Brazil, a continent-sized country with very heterogeneous socioeconomic realities and an uneven distribution of ophthalmologists [17], home to the fifth biggest diabetic population in the world [18] and also to the largest public free and chronically underfinanced healthcare system [19]. Multiple socioeconomic barriers prevent access to eye examination in the poor regions of Brazil [3]. Recently, several authors have proposed teleophthalmology to increase access in Brazil and elsewhere, with favorable cost-effectiveness results and a reduced burden to specialized services $[3,16,17]$, in both geographically isolated [15] and highly urbanized areas [20].

In the present study, we propose a decision tree based not only on DR grading, but also on the presence of ocular media opacities. Systematic evaluation of the anterior segment is not universally performed in teleophthalmology strategies of DR screening [15, 16], and the presence of cataracts is usually considered a flaw in such strategies, as lens opacities frequently preclude DR classification [21]; some screening protocols propose the exclusion of patients with cataracts [22]. However, by evaluating anterior segment images, the reader is able to judge whether cataracts are the reason for ungradable fundus images, which is a clinically relevant information, as cataracts are a significant public health issue and an important cause of blindness in 
developing countries [23]. We believe that grouping cataract and referable DR patients is effective in providing proper health care for those individuals; cataract referral and treatment in patients with diabetes should follow a special protocol because of the increased risk of ocular complications in such patients.

The patients enrolled for diabetic retinopathy screening with a portable retinal camera adequately represent the population with DM2 treated in primary health care, characterized by the predominance of the elderly, mostly women, diabetes duration over 10 years and regular glycemic control. Arterial hypertension and dyslipidemia were the most prevalent comorbidities associated with DM [24]. We observed that patients with referable DR had higher HbA1c than those with DR absent or non-referable, while other clinical and demographic variables were similar. The association between poor glycemic control and severity and progression of retinopathy is well established in the literature, in addition to diabetes duration, nephropathy, hypertension and dyslipidemia [8]. Further, DR has been related to the development of macrovascular complications of diabetes, specifically, cerebrovascular, cardiovascular and peripheral complications [25]. Our results also showed that the imaging protocol had a poorer diagnostic yield in older patients, who have also experienced a longer duration of the examination. Ocular characteristics such as impaired pupil dilation may have played a role, even though the images acquisition has been done after pharmacological mydriasis.

This study's strengths are its "real-life" approach, since it was performed during ongoing health care by a team of nurses in a primary care setting, and the feasibility of a protocol that involves a handheld device and a telemedicine approach, offering the perspective of DR screening with mobile units and home evaluation. Such strategy potentially increases access in underserved areas, concomitantly offering a safer alternative to individuals with diabetes, considering the vulnerability brought by the ongoing COVID-19 pandemic: Mobile units can ultimately reduce the number of patients' travels and contacts; furthermore, image acquisition with such devices is compatible with physically distancing between the patient and the operator and may even be performed outdoors. Regarding study limitations, it should be pointed out that only patients who dwell in an urban area were evaluated; furthermore, other ophthalmological evaluation modalities, such as visual acuity measurement, slitlamp examination or optical coherence tomography, were not available, thus limiting the conclusions on diabetic maculopathy.

\section{Conclusion}

Our data point to the feasibility of a low-cost DR screening strategy which involves training of non-specialized healthcare personnel, a handheld device and telemedicine. Such protocol is compatible with the Family Health Strategy, with the potential to increase the coverage of DR screening in underserved areas; the possibility of mobile units is also relevant as an alternative to clinical examination for DR screening in the context of the COVID-19 pandemic. A major challenge is to provide timely treatment for detected cases of sight-threatening DR and cataract.

Acknowledgements The authors thank Phelcom Technologies, São Carlos, Brazil, for making available the smartphone-based handheld device and the EyerCloud platform.

Authors' contribution statement MSQ and FKM conceived and designed the study, oversaw the study implementation and collection of data, interpreted the data, contributed to the writing of the first draft of the manuscript and its subsequent revisions and contributed to its intellectual content. Both of them are the guarantor of this work and, as such, had full access to all data in the study and take responsibility for the integrity of data and the accuracy of the data analyses; JXC collected the data and oversaw the implementation of the study protocol; SFB, MRM, CGDC and EASA collected the data; and MLCG oversaw the study implementation and collection of data and contributed to its intellectual content.

Funding This research did not receive any specific grant from any funding agency in the public, commercial or not-for-profit sector.

Availability of data and material The datasets generated during and/or analyzed during the current study are available from the corresponding author on reasonable request.

\section{Compliance with ethical standards}

Conflict of interest The authors declare that they have no conflict of interest.

Ethics approval This study was performed in line with the principles of the Declaration of Helsinki. Approval was granted by the Ethics Committee of Universidade Nove de Julho (\#3050387 and \#3141417).

Informed consent Informed consent was obtained from all individual participants included in the study.

\section{References}

1. Davila JR, Sengupta SS, Niziol LM, Sindal MD, Besirli CG, Upadhyaya $S$ et al (2017) Predictors of photographic quality with a handheld nonmydriatic fundus camera used for screening of vision-threatening diabetic retinopathy. Ophthalmologica 238(12):89-99. https://doi.org/10.1159/000475773

2. Flaxel CJ, Adelman RA, Bailey ST, Lim JI, Vemulakonda GA, Ying G et al (2020) Diabetic retinopathy preferred practice 
pattern. Ophthalmology 127(1):66-145. https://doi.org/10.1016/j. ophtha.2019.09.025

3. Ben ÂJ, Neyeloff JL, de Souza CF, Rosses APO, de Araujo AL, Szortika A et al (2020) Cost-utility analysis of opportunistic and systematic diabetic retinopathy screening strategies from the perspective of the Brazilian public healthcare system. Appl Health Econ Hea 18(1):57-68. https://doi.org/10.1007/s40258-01900528-w

4. Macinko J, Harris MJ (2015) Brazil's family health strategy: delivering community-based primary care in a universal health system. N Engl J Med 372(23):2177-2181. https://doi.org/10.1056/ NEJMp1501140

5. Salongcay RP, Silva PS (2019) The role of teleophthalmology in the management of diabetic retinopathy. Asia Pac J Ophthalmol. https://doi.org/10.22608/APO.2017479

6. Kalogeropoulos D, Kalogeropoulos C, Stefaniotou M, Neofytou M (2020) The role of tele-ophthalmology in diabetic retinopathy screening. J Optom. https://doi.org/10.1016/j.optom.2019.12.004

7. Malerbi FK, Morales PH, Farah ME, Drummond KRG, Mattos TCL, Pinheiro AA et al (2015) Comparison between binocular indirect ophthalmoscopy and digital retinography for diabetic retinopathy screening: the multicenter Brazilian Type 1 Diabetes Study. Diabetol Metab Syndr 7(1):116. https://doi.org/10.1186/ s13098-015-0110-8

8. American Diabetes Association (2020) Microvascular complications and foot care: standards of medical care in diabetes. Diabetes Care 43(1):135-151. https://doi.org/10.2337/dc20-s011

9. Liew G, Michaelides M, Bunce C (2014) A comparison of the causes of blindness certifications in England and Wales in working age adults (16-64 years), 1999-2000 with 2009-2010. BMJ Open 4(2):e004015. https://doi.org/10.1136/bmjopen-2013-00401 5

10. Forster AS, Forbes A, Dodhia H, Connor C, Du Chemin A, Sivaprasad S et al (2013) Changes in detection of retinopathy in type 2 diabetes in the first 4 years of a population-based diabetic eye screening program. Diabetes Care 36(9):2663-2669. https:// doi.org/10.2337/dc13-0130

11. Looker HC, Nyangoma SO, Cromie DT, Olson JA, Leese GP, Black MW et al (2014) Rates of referable eye disease in the Scottish National Diabetic Retinopathy Screening Programme. Brit J Ophthalmol 98(6):790-795. https://doi.org/10.1136/bjophthalm ol-2013-303948

12. Hautala N, Aikkila R, Korpelainen J, Keskitalo A, Kurikka A, Falck A et al (2014) Marked reductions in visual impairment due to diabetic retinopathy achieved by efficient screening and timely treatment. Acta Ophthalmol 92(6):582-587. https://doi. org/10.1111/aos.12278

13. Scanlon PH (2017) The English National Screening Programme for diabetic retinopathy 2003-2016. Acta Diabetol 54:515-525. https://doi.org/10.1007/s00592-017-0974-1

14. Li HK, Horton M, Bursell SE, Cavallerano J, Zimmer-Galler I, Tennant $\mathrm{M}$ et al (2011) Telehealth practice recommendations for diabetic retinopathy. Second edition. Telemed E-Health 17(10):814-837. https://doi.org/10.1089/tmj.2011.0075

15. Kanjee R, Dookeran RI, Mathen MK, Stockl FA, Leicht R (2017) Six-year prevalence and incidence of diabetic retinopathy and cost-effectiveness of tele-ophthalmology in Manitoba. Can J Ophthalmol 52(Suppl 1):S15-S18. https://doi.org/10.1016/j. jcjo.2017.09.022

16. Souza GF, Figueira RM, Alkmim MB, Sousa LAP, Bonisson L, Ribeiro ALP et al (2020) Teleophthalmology screening for diabetic retinopathy in Brazil: applicability and economic assessment. Telemed J E Health 26(3):341-346. https://doi.org/10.1089/ tmj.2018.0241

17. Malerbi FK, Matsudo NH, Carneiro ABM, Lottenberg CL (2015) Retinal diseases in a reference center from a Western Amazon capital city. Einstein (São Paulo) 13(4):530-534. https://doi. org/10.1590/S1679-45082015AO3538

18. Saeedi P, Petersohn I, Salpea P, Malanda B, Karuranga S, Unwin $\mathrm{N}$ et al (2019) Global and regional diabetes prevalence estimates for 2019 and projections for 2030 and 2045: results from the International Diabetes Federation Diabetes Atlas, 9th edition. Diabetes Res Clin Pract 157:107843. https://doi.org/10.1016/j. diabres.2019.107843

19. Pasternak J (2018) What is the future of the Brazilian Public Health System? Einstein 16(4):1. https://doi.org/10.31744/einst ein_journal/2018ED4811

20. Sim DA, Mitry D, Alexander P, Mapani A, Goverdhan S, Aslam T et al (2016) The evolution of teleophthalmology programs in the United Kingdom: beyond diabetic retinopathy screening. J Diabetes Sci Technol 10(2):308-317. https://doi.org/10.1177/19322 96816629983

21. Rani PK, Bhattarai Y, Sheeladevi S, Shiva Vaishnavi K, Ali BJ (2018) Analysis of yield of retinal imaging in a rural diabetes eye care model. Indian J Ophthalmol 66(2):233-237. https://doi. org/10.4103/ijo.IJO_500_17

22. Jamali S, Abrishami M, Lashay A, Ashrafi E, Adibi H, Ghaderi E et al (2020) Comparison of portable cameras for diabetic retinopathy community screening. J Diabetes Sci Technol. https://doi. org/10.1177/1932296820929357

23. Furtado JM, Berezovsky A, Ferraz NN, Muñoz S, Fernandes AG, Watanabe SS et al (2019) Prevalence and causes of visual impairment and blindness in adults aged 45 years and older from Parintins: the Brazilian Amazon region eye survey. Ophthalmic Epidemiol 26(5):345-354. https://doi.org/10.1080/09286 586.2019.1624783

24. Coutinho WF, Silva WS Jr (2015) Diabetes care in Brazil. Ann Glob Health 81(6):735-741. https://doi.org/10.1016/j. aogh.2015.12.010

25. Pearce I, Simó R, Lövestam-Adrian M, Wong DT, Evans M (2019) Association between diabetic eye disease and other complications of diabetes: implications for care. A systematic review. Diabetes Obes Metab 21(3):467-478. https://doi.org/10.1111/dom.13550

Publisher's Note Springer Nature remains neutral with regard to jurisdictional claims in published maps and institutional affiliations. 